\section{Inhibit integrins to protect cartilage}

Integrins link chondrocytes to the surrounding extracellular matrix (ECM), mediating chondrocyte development and differentiation and controlling catabolic pathways that cause cartilage destruction. New research has revealed a role for integrin $\beta$-like protein 1 (ITGBL1) in modulating integrin-mediated chondrocyte activity, suggesting that blocking integrins with ITGBL1 could reduce cartilage damage in osteoarthritis (OA).

"ITGBL1 is a secreted protein that possesses a dual function in inhibiting inflammation and promoting chondrogenesis," explains corresponding author Tae Joo Park. "Our data suggest that ITGBL1 might function as a secreted inhibitor for a broad range of integrins."

Fragments of ECM molecules such as fibronectin are produced via proteolytic cleavage of the ECM by catabolic enzymes during cartilage degeneration. These fragmented
ECM molecules can themselves trigger the production of catabolic enzymes by chondrocytes in a positive feedback loop. "Integrins are known to be receptors for fragmented ECM molecules and to promote chondrolysis in models of arthritis," says Park.

In vitro studies showed that overexpression of ITGBL1 reduced the binding of fragmented ECM molecules to human chondrocytes via integrins. Similarly, stimulation of human chondrocytes with fragmented ECM molecules caused an upregulation of catabolic enzymes that could be reduced by the overexpression of ITGBL1.

In mice, knockdown of Itgbl1 using intra-articular adenoviral delivery of short hairpin RNA caused cartilage damage similar to that seen in patients with OA. Interestingly, expression of ITGBL1 was also greatly reduced in cartilage

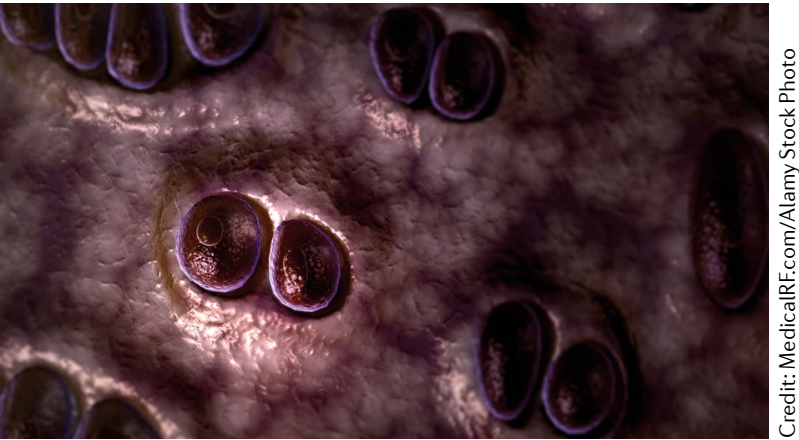

from patients with OA compared with undamaged control cartilage. Furthermore, in the destabilization of the medial meniscus mouse model of OA, intra-articular adenoviral delivery of Itgbl1 reduced cartilage damage and osteophyte formation.

"As a long-term goal, we are trying to identify the minimal domain of ITGBL1 that could be used to treat various integrin-mediated diseases," states Park. "Ultimately, we hope to develop peptide drugs based on the ITGBL1 sequence."

Joanna Collison osteophyte formation
ORIGINAL ARTICLE Song, E. K. et al. ITGBL1 modulates integrin activity to promote cartilage formation and protect against arthritis. Sci. Transl. Med. 10, eaam7486 (2018)

\title{
A new antisense oligonucleotide therapy?
}

Current treatments for osteoarthritis (OA) can alleviate the symptoms of joint pain, but do not modify the course of disease. One strategy to overcome this therapeutic limitation is to target the underlying pathogenic mechanisms that degrade the cartilage. New research demonstrates that injection of miR-181a-5p antisense oligonucleotides might be a suitable therapeutic method to achieve this goal.

"Three years ago, we conducted a screen for microRNAs in patients with varying degrees of spinal facet joint cartilage degeneration," explains Mohit Kapoor, corresponding author of the new study. "Our results showed that miR-181a-5p has an active role in degenerating facet cartilage."

In this follow-up work, Kapoor and his team tested locked nucleic acid antisense oligonucleotides that can

target miR-181a-5p from mice, rats and humans. They show that these

intra-articular injection of the oligonucleotides can protect rats from cartilage degradation

5
oligonucleotides limit expression of catabolic and apoptotic markers by murine and human cartilage cells in response to IL-1 $\beta$ and in cultured knee cartilage explants from patients with $O A$.

In support of these findings, the researchers show that intra-articular injection of the oligonucleotides can protect rats from cartilage degradation (measured by OARSI scores) in an OA model driven by needle puncture of the facet joint.

Similar results were obtained in mice using a different OA model, by surgical destabilization of the medial meniscus. In this model, reduction in cartilage destruction resulted from the oligonucleotides being injected into the joints 2 or 4 weeks after surgery to induce OA. This time frame might indicate that

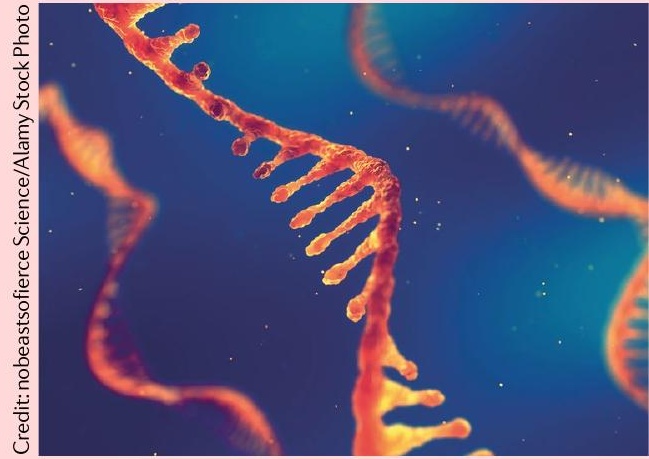

the effect of this method has therapeutic (not just prophylactic) potential.

"We are now conducting a series of studies to investigate the safety and best therapeutic dose, as well as optimal method for the local delivery of miR-181a-5p antisense oligonucleotides to the joints," says Kapoor. "If the oligonucleotides prove to be safe and effective, this could be a potential OA disease modifying therapy and not just a symptom modifier," he concludes.

Nicholas J. Bernard

ORIGINAL ARTICLE Nakamura, A. et al.

microRNA-181a-5p antisense oligonucleotides attenuate osteoarthritis in facet and knee joints. Ann. Rheum. Dis. https://doi.org/10.1136/ annrheumdis-2018-213629 (2018) 